\title{
Modeling and Simulation of Flow and Formation Damage of Asphalt-Paved Roads
}

\author{
M. H. Alawi, ${ }^{1}$ M. M. El-Qadi, ${ }^{2}$ and M. A. El-Ameen ${ }^{3}$ \\ ${ }^{1}$ Civil Engineering Department, Collage of Engineering, Umm Al-Qura University, Saudi Arabia \\ ${ }^{2}$ Mathematics Department, Faculty of Science, Helwan University, Egypt \\ ${ }^{3}$ Preparing Year Department, Collage of Engineering, Umm Al-Qura University, Saudi Arabia
}

Correspondence should be addressed to M. A. El-Ameen; mohamedmossa327@yahoo.com

Received 29 September 2013; Accepted 5 October 2013

Academic Editor: Mohamed Fathy El-Amin

Copyright (C) 2013 M. H. Alawi et al. This is an open access article distributed under the Creative Commons Attribution License, which permits unrestricted use, distribution, and reproduction in any medium, provided the original work is properly cited.

\begin{abstract}
Porous asphalt is a standard asphalt built on aggregate storage bed which allows water to drain through it and reduces stormwater runoff. However, porosity of the porous asphalt and the storage bed may be effectively reduced due to trapping suspended solids from the water or from the asphalt damage. In this paper, we present mathematical modeling and numerical simulation of flow and damage of porous asphalt-paved roads. A mathematical model to describe the fine-particles transport carried by a two-phase flow in a porous medium is presented. The buoyancy, capillarity, and mixed relative permeabilities correlations to fit with the mixed-wet system are considered. Throughout this investigation, we monitor the changing of the fluids properties such as water saturation and solid properties such as porosity and permeability due to trapping the fine-particles.
\end{abstract}

\section{Introduction}

The impervious asphalt-paved roads may store significant amounts of thermal energy during summer. The stored thermal energy may be transferred to stream waters during runoff events. Moreover, as a result of fluctuations in ambient air temperatures-diurnal and seasonal, intensity of solar radiation of the asphalt-paved, a significant deformation of asphalt-paved may occur due to the heat and mass transfer between asphalt and water. On the other hand porous (pervious, permeable, or open-graded) asphalt is standard asphalt with reduced sand or fines that allows water to drain through it. Pervious asphalt built on aggregate storage bed reduces stormwater runoff. In addition to reducing runoff, this effectively traps suspended solids and filters pollutants from the water. The stormwater flows through the asphalt to the layer of crushed stone aggregate bedding and base that supports the asphalt while providing storage and runoff treatment. The use of porous asphalt can potentially reduce additional expenditures and land consumption for conventional collection, conveyance, and detention stormwater infrastructure. Compared to the lifetime of dense graded asphalt concrete roads, the lifetime of porous asphalt concrete is less [1]. The loss of stones from the road surface, called raveling, is mostly reported as the dominant defect in porous asphalt wearing course $[2,3]$. Raveling is the start of major defects like potholes, because once a stone is gone, the surrounding stones will follow for lacking support in at least one direction [4]. Raveling also has negative influence on the noise reduction function and skid resistance of porous asphalt pavement. During service life, the pores tend to be clogged by dirt, dust, or other clogging agents. The formulation of fine-particles transport in two-phase flow in porous media has been studied experimentally and numerically in [5-7]. Formation damage is a common problem in reservoir development. The particles migrate through the porous media, deposit on the pore surfaces, and become trapped at pore constrictions to reduce the rock porosity and permeability.

Researchers have done many attempts to predict these phenomena experimentally and numerically; however, there is no study that considers the possible porosity and permeability reduction. So, in order to keep porous asphalt and its storage bed efficient, considering these kinds of effects may 
lead to interesting results. In the current work, we introduce modeling and numerical simulation of fine-particle transport in two-phase flow in porous asphalt-paved roads.

\section{Mathematical Modeling}

The basic equations that govern the flow of the two-phase flow in porous media are mass conservation equation and constitutive equation (Darcy's law). The two-dimensional governing equations may be written as

$$
\begin{gathered}
\frac{\partial\left(\varphi \rho_{w} S_{w}\right)}{\partial t}=-\nabla \cdot\left(\rho_{w} v_{w}\right), \\
\frac{\partial\left(\varphi \rho_{a} S_{a}\right)}{\partial t}=-\nabla \cdot\left(\rho_{a} v_{a}\right), \\
v_{w}=-\frac{K k_{r w}}{\mu_{w}}\left(\nabla p_{w}-\rho_{w} \mathbf{g}\right), \\
v_{a}=-\frac{K k_{r a}}{\mu_{a}}\left(\nabla p_{a}-\rho_{a} \mathbf{g}\right),
\end{gathered}
$$

where $S$ is the saturation and $v[\mathrm{~m} / \mathrm{s}]$ is the velocity. $w$ stands for the wetting phase (water), and $a$ stands for the nonwetting phase (air). $\varphi$ is the porosity of the medium, and $\nabla=(\partial / \partial x, \partial / \partial z)$ is the divergence operator. $K\left[\mathrm{~m}^{2}\right]$ is the absolute permeability, $k_{r \alpha}$ [dimensionless] is the relative permeability, $\rho\left[\mathrm{kg} \mathrm{m}^{3}\right]$ is the density, $p[\mathrm{~Pa}]$ is the pressure, and $\mathbf{g}=(0,-g)^{T}$ is the gravitational acceleration. $\mu$ is the viscosity. The fluid saturations for the two-phase flow are interrelated by

$$
S_{w}+S_{a}=1
$$

The total velocity is

$$
v_{t}=v_{w}+v_{a}
$$

Summing the saturation equation for water phase and the oil phase, one obtains

$$
\nabla \cdot v_{t}=0
$$

Moreover, adding the constitutive equations for each phase, (1a)-(2b), and substituting into (5), we end up with

$$
v_{t}=-K\left[\lambda_{t}(S) \nabla p_{w}+\lambda_{a}(S) \nabla p_{c}\right]+K \chi(S) g,
$$

where $\lambda_{\alpha}(S)=k_{r \alpha}(S) / \mu_{\alpha}$ is the mobility, $\lambda_{t}(S)=\lambda_{a}(S)+\lambda_{w}(S)$ is the total mobility, and $\chi=\lambda_{a}(S) \rho_{a}+\lambda_{w}(S) \rho_{w}$. In order to derive the pressure equation, substitute (6) into (5). We obtain

$$
\nabla \cdot\left[\lambda_{t}(S) \nabla p_{w}+\lambda_{a}(S) \nabla p_{c}(S)-\chi(S) g\right]=0 .
$$

Substituting the constitutive equation of the water phase, (2a) and (2b), into (1a) and (1b) gives

$$
\frac{\partial\left(\varphi S_{w}\right)}{\partial t}+\nabla \cdot v_{w}=0
$$

Therefore, water velocity may be written as

$$
v_{w}=f_{a} v_{t}+\lambda_{w} K\left[f_{a} \nabla p_{c}\right]+\lambda_{w} f_{a} K \Delta \rho g,
$$

where $\Delta \rho=\rho_{w}-\rho_{a}$.

On the other hand, a mathematical model is developed to describe the fine-particles transport carried by two-phase flow in porous media. Assuming that we have a number $m$ of size intervals of particles in the water phase, the transport equation for each size interval $i$ of the particles in the water phase can be written as

$$
\frac{\partial\left(\phi S_{w} C_{i}\right)}{\partial t}+v_{w} \cdot \nabla C_{i}=\nabla \cdot\left(\varphi S_{w} D_{i} \nabla C_{i}\right)+R_{i},
$$

where $i=1,2, \ldots, m . D_{i}$ is the dispersion coefficients of particles in size interval $i$ in the water phase. $R_{i}$ is the net rate of loss of particles in size interval $i$ in the water phase. The net rate of loss of particles may be written as [5-7] follows:

$$
R_{i}=\frac{\partial(\delta \phi)_{i}}{\partial t}
$$

where $(\delta \phi)_{i}=v_{i}+v_{i}^{*}$ is the porosity change due to release or retention of particles of interval $i$ in the water phase. $v_{i}$ is the volume of the particles of interval size $i$ in contact with the water phase available on the pore surfaces per unit bulk volume of sandstone. $v_{i}^{*}$ is the volume of the particles of interval size $i$ entrapped in pore throats from the water phase per unit bulk volume of sandstone due to plugging and bridging. At the critical velocity of the surface deposition only particle retention occurs while above it retention and entrainment of the particles take place simultaneously [8]. A modified Gruesbeck and Collins's model for the surface deposition is expressed by [5-7] the following:

$$
\frac{\partial v_{i}}{\partial t}= \begin{cases}\alpha_{d, i}\left|v_{w}\right| C_{i} & \text { when } v_{w} \leq v_{c} \\ \alpha_{d, i}\left|v_{w}\right| C_{i}-\alpha_{e, i} v_{i}\left|v_{w}-v_{c}\right| & \text { when } v_{w}>v_{c}\end{cases}
$$

where $\alpha_{d, i}$ is the rate coefficients for surface retention of the particles in interval i. $\alpha_{e, i}$ is the rate coefficients for entrainment of the particles in interval $i . v_{c}$ is the critical velocity. Similarly, the rate of entrapment of the particles in interval $i$ is

$$
\frac{\partial v_{i}^{*}}{\partial t}=\alpha_{p t, i}\left|v_{w}\right| C_{i}
$$

where $\alpha_{p t, i}$ is the pore throat blocking constants. Porosity may be changed because of particles deposition on the pore surfaces or blocking of pore throats. The porosity variation may be expressed by [5-7] the following:

$$
\phi=\phi_{0}-(\delta \phi)_{i}
$$

where $\phi_{0}$ is the initial porosity. Also, the permeability variation due to particles deposition on the pore surfaces or blocking of pore throats may be expressed as [5-7] follows:

$$
K=K_{0}\left((1-f) k_{f}+\frac{f \phi}{\phi_{0}}\right)^{l}
$$


where $K_{0}$ is the initial permeability, $k_{f}$ is a constant for fluid seepage allowed by the plugged pores, $f=1-$ $\sum_{i} \alpha_{f, i} v_{i}^{*}$ is the fraction of the original cross-sectional area open to flow, and the value of the exponent $l$ has range from 2.5 to 3.5 . For the particles transport carried by fluid stream in the porous media, deposition on pore surfaces and blockage in pore throats may occur. The retained particles on pore surfaces may desorb for hydrodynamic forces and then possibly adsorb on other sites of the pore bodies or get entrapped at other pore throats.

\section{Results and Discussion}

In order to get physical insights for the problem under consideration, we consider that typical cross-section for pervious pavement system consists of six porous layers (Figure 1) that can be simplified to one-dimensional system for calculations. The porous asphalt layer taken is $15 \mathrm{~cm}$, the choker course layer is $20 \mathrm{~cm}$, the filter course is $30 \mathrm{~cm}$, the filter blanket is $8 \mathrm{~cm}$, the reservoir course is $10 \mathrm{~cm}$, and finally the native material is optional that we consider it $20 \mathrm{~cm}$. We consider the top boundary as inlet of water-particles suspension. So water imbibes into the porous asphalt and replaces the air that leaves the medium from the same boundary. This type of flow in porous media is called countercurrent imbibition [9-12]. In countercurrent imbibition both wetting and nonwetting phases flow through one inflow-outflow boundary. Therefore, the total velocity becomes zero, $v_{t}=0$. Therefore, the flow equations may be written as

$$
\begin{aligned}
\frac{\partial P_{w}}{\partial z} & =-f_{w}(S) \frac{\partial P_{c}(S)}{\partial z}-\chi(S) g, \\
v_{w} & =K \lambda_{w} f_{a}\left(\frac{\partial P_{c}}{\partial z}+\Delta \rho g\right) .
\end{aligned}
$$

Therefore,

$$
\frac{\partial\left(\varphi S_{w}\right)}{\partial t}+K \frac{\partial}{\partial z}\left[\lambda_{w} f_{a}\left(\frac{\partial P_{c}}{\partial z}-\Delta \rho g\right)\right]=0 .
$$

The relative permeabilities are given in terms of the normalized wetting phase saturation $S$, given as

$$
\begin{gathered}
k_{r w}=k_{r w}^{0} S^{a}, \\
k_{r a}=k_{r a}^{0}(1-S)^{b},
\end{gathered}
$$

where $a$ and $b$ are positive numbers, $k_{r w}^{0}=k_{r w}(S=1)$ is the endpoint relative permeability to wetting phase, and $k_{r a}^{0}=k_{r a}(S=0)$ is the endpoint relative permeability to nonwetting phase. The normalized wetting phase saturation is given by

$$
S=\frac{S_{w}-S_{i w}}{1-S_{r a}-S_{i w}}, \quad 0 \leq S \leq 1,
$$

where $S_{i w}$ is the irreducible water saturation and $S_{r a}$ is the residual air saturation.

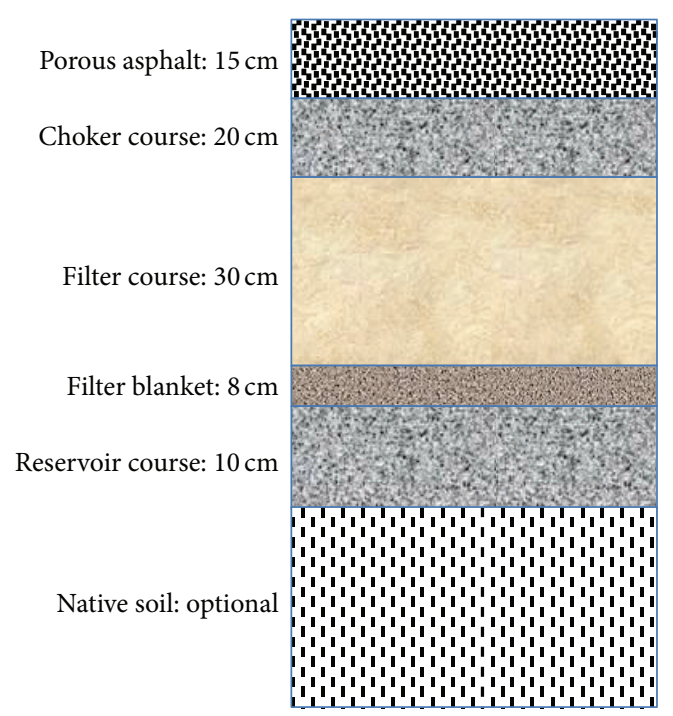

Figure 1: Typical cross-section for pervious pavement system.

For the capillary pressure we used the general correlation

$$
p_{c}=c_{w}\left(\frac{S_{w}-S_{w r}}{1-S_{w r}}\right)^{-a_{w}}+c_{a}\left(\frac{S_{a}-S_{a r}}{1-S_{a r}}\right)^{-a_{a}},
$$

where $c_{w}$ and $c_{a}$ are constants that represent the entry pressure for imbibition and drainage, respectively. The constants $1 / a_{w}$ and $1 / a_{a}$ are the pore size distribution indexex for imbibition and drainage, respectively.

The transport equation for the interval $i$ of the particles can be written as

$$
\frac{\partial\left(\varphi S_{w} C_{i}\right)}{\partial t}+v_{w} \frac{\partial C_{i}}{\partial z}=\frac{\partial}{\partial z}\left(\varphi S_{w} D_{i} \frac{\partial C_{i}}{\partial z}\right)+R_{i},
$$

$R_{i}$

$$
= \begin{cases}\left(\alpha_{d, i}+\alpha_{p t, i}\right)\left\|v_{w}\right\| C_{i}, & \left\|u_{w}\right\| \leq u_{c} \\ \left(\alpha_{d, i}+\alpha_{p t, i}\right)\left\|v_{w}\right\| C_{i}-\alpha_{e, i} v_{i}\left\|v_{w}-v_{c}\right\| C_{i}, & \left\|u_{w}\right\|>u_{c} .\end{cases}
$$

The surface deposition of the particles in the interval $i$ is

$$
\frac{\partial v_{i}}{\partial t}= \begin{cases}\alpha_{d, i}\left\|v_{w}\right\| C_{i}, & \left\|v_{w}\right\| \leq u_{c} \\ \alpha_{d, i}\left\|v_{w}\right\| C_{i}-\alpha_{e, i} v_{i}\left\|v_{w}-v_{c}\right\| C_{i}, & \left\|v_{w}\right\|>u_{c} .\end{cases}
$$

The rate of entrapment of the particles in interval $i$ is

$$
\frac{\partial v_{i}^{*}}{\partial t}=\alpha_{p t, i}\left\|v_{w}\right\| C_{i}
$$

The initial conditions are

$$
\begin{array}{r}
S_{w}=S_{0 w}, \quad C_{i}=v_{i}=v_{i}^{*}=0, \\
t=0, \quad 0 \leq z \leq H,
\end{array}
$$




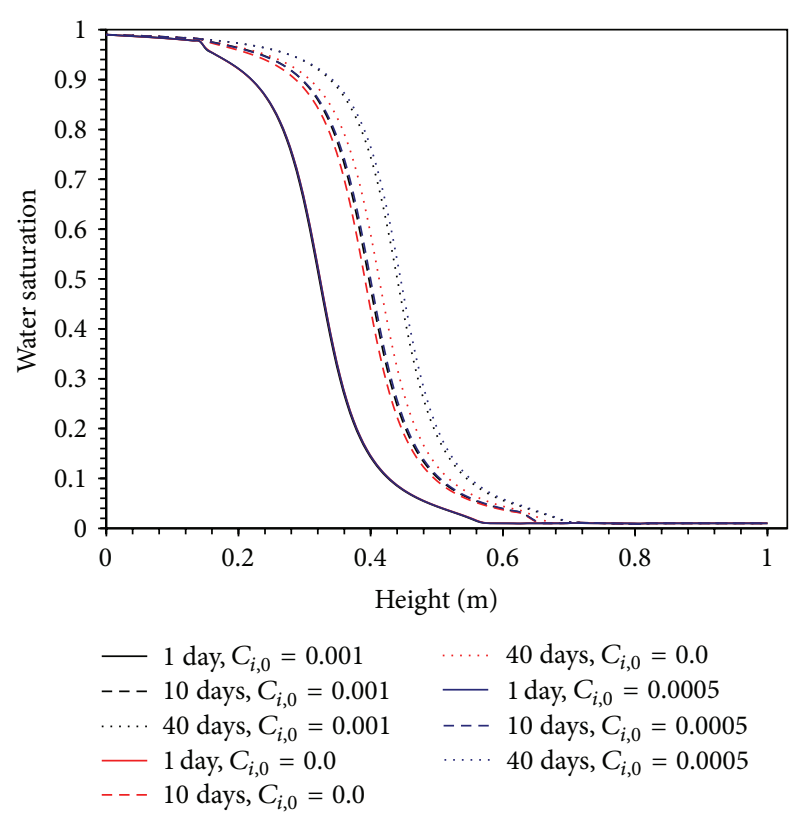

FIGURE 2: Water saturation against the height with various imbibition times and inlet concentrations.

where $H$ is the depth and $S_{0 w}$ is the initial water saturation. The boundary conditions are

$$
\begin{gathered}
S_{w}=1-S_{\text {or }}, \quad C_{i}=C_{i, 0}, \quad v_{i}=v_{i}^{*}=0, \\
t \geq 0, \quad z=0, \\
\frac{d S_{w}}{d z}=\frac{d C_{i}}{d z}=\frac{d v_{i}}{d z}=\frac{d v_{i}^{*}}{d z}=0, \\
t \geq 0, \quad z=H,
\end{gathered}
$$

where $C_{i, 0}$ is the concentration of particles in the particles suspension at the inlet boundary. The governing equations (17)-(23) are solved numerically along with the initial and boundary conditions, (24)-(25). An efficient algorithm is used to solve the above high-nonlinear parabolic partial differential equation in one space variable $z$ and time $t$. The Galerkin method is used for spatial discretization [13], while the time integration for the resulting ordinary differential equation is done with an adaptive time step. 100 points of the spatial grid were used during calculations and were enough to provide an acceptable accuracy. Now, we consider one-size particles suspension in the water phase at the inlet, with the following parameter [6,7], $\alpha_{d, i}=8 \mathrm{~m}^{-1}, \alpha_{p t, i}=17 \mathrm{~m}^{-1}, \alpha_{e, i}=$ $400 \mathrm{~m}^{-1}, v_{c}=4.6 \times 10^{-3} \mathrm{~m} / \mathrm{s}$, and $D_{i}=5.6 \times 10^{-3} \mathrm{~m}^{2} / \mathrm{s}$. The inlet particles concentration values are $C_{i, 0}=0.0$ (without particles), 0.0005 , and 0.01 . The remaining model parameters are $S_{w r}=S_{o r}=0.001, a_{w}=a_{a}=0.5, c_{w}=500, c_{a}=-500$, $\varphi_{0}=0.2, l=3, k_{f}=0.015, \gamma_{f}=0.01, k_{r w 0}=k_{r a 0}=1$, and $a=b=4$.

Figure 2 shows the water saturation against the dimensionless distance with various imbibition times and inlet particles concentrations. It is notable that the concentration of particles increases the water saturation in particular after

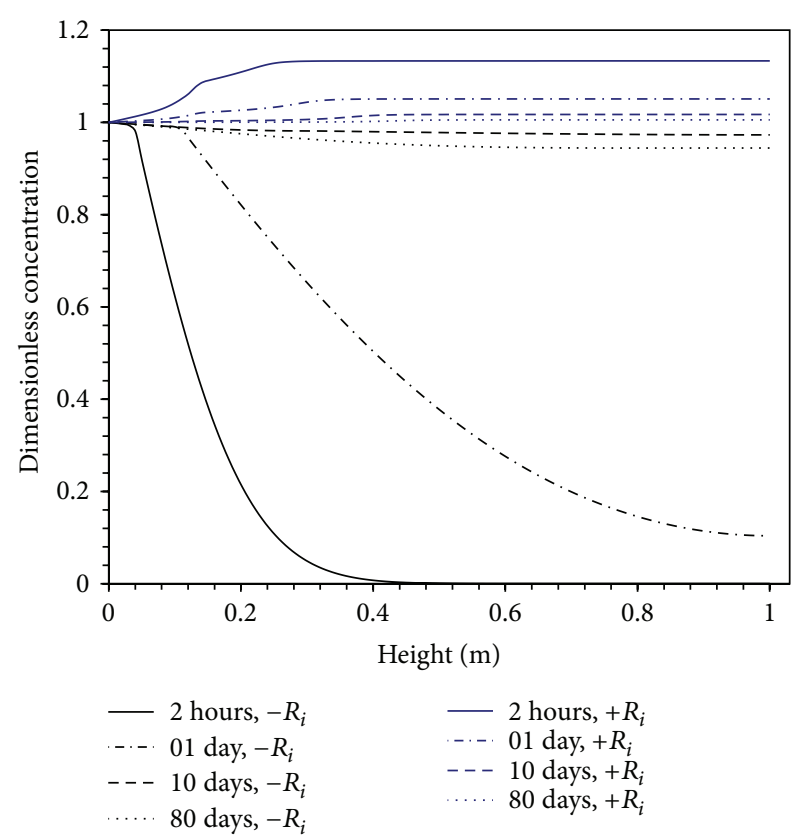

FIgURE 3: Normalized particles concentration against height with positive/minus $R_{i}$ and various imbibition times.

significant time of imbibition. Moreover, the saturation of water increases with the time imbibition.

The normalized particles concentration is plotted in Figure 3 against the dimensionless distance with various inlet concentrations and imbibition times. From this figure it can be seen that the concentration of particles increases as the concentration at the inlet increases for positive $R_{i}$. An interesting phenomenon can be observed from Figure 3 that the concentration increases with time but after a certain time of imbibition concentration of particles start to decrease with time. This may be interpreted by increasing the rate of particles precipitation on the pore surface of the porous medium. The opposite is true for minus $R_{i}$. It is noteworthy that, in Figure 3, the dimensionless concentration goes larger than 1 because the concentration of particles increases by erosion of more particles from the medium $\left(-R_{i}\right)$. On the other hand, the reference concentration was the inlet concentration of the particles coming with water from outside.

Figures 4 and 5 illustrate the ratios of the permeability and porosity against the dimensionless distance with various imbibition times, respectively. Both the permeability and the porosity are reduced due to the precipitation of the particles on the pores walls. It is interesting to note that the reduction rates of the permeability and porosity depend on the layer permeability of the medium.

\section{Conclusions}

In this pape, we presented numerical modeling and simulation of particle transport in two-phase flow in porous asphaltpaved roads. Numerical experiments have been performed to explore these phenomena and to study the possible porosity and permeability variations. We found that both 


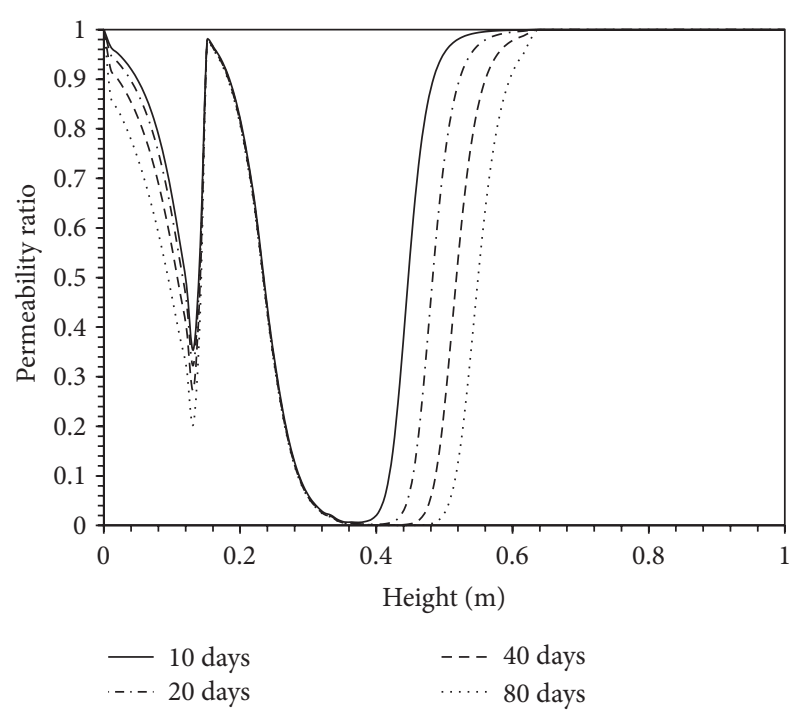

FIGURE 4: Permeability ratio against the height with various imbibition times.

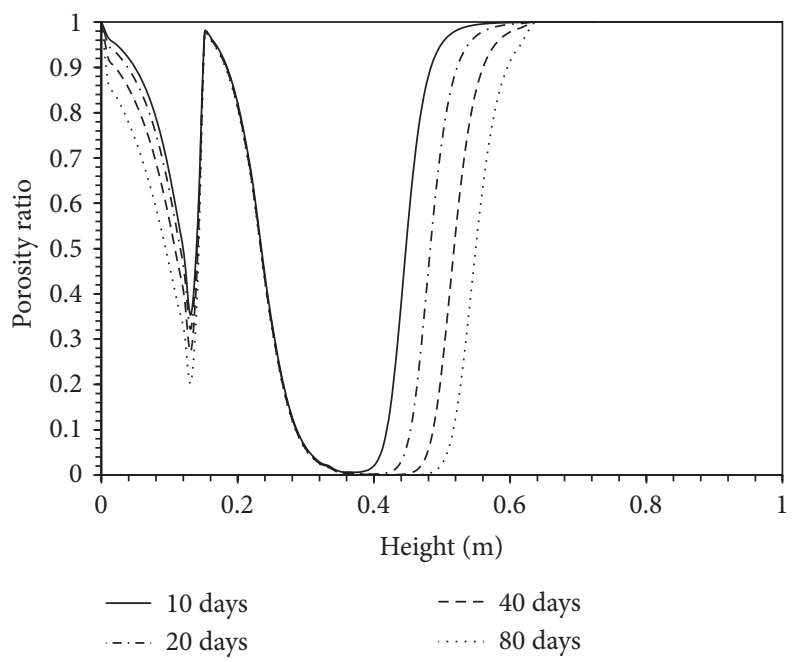

FIGURE 5: Porosity ratio against the height with various imbibition times.

the permeability and the porosity are reduced due to the precipitation of the particles on the pores walls. These results may help engineers to keep porous asphalt and its storage bed efficient by looking for cleaning methodologies to avoid media blocking.

\section{Acknowledgment}

The authors would like to thank the Institute of Scientific Research and Revival of Islamic Heritage, Umm Al-Qura University, for supporting the Project no. 43208015.

\section{References}

[1] J. L. M. Voskuilen and P. N. W. Verhoef, "Cause of premature ravelling failure of porous asphalt," in Proceedings of the 6th International RILEM Symposium on Performance Testing and Evaluation of Bituminous Materials, pp. 191-197, 2003.
[2] C. Padmos, "Over ten years experience with porous road surfaces," in Proceedings of the ISAP 9th International Conference on Asphalt Pavements, Copenhagen, Denmark, 2002.

[3] J. L. M. Voskuilen and M. Huurman, Conversations, Centre For Transport and Navigation of the Dutch Ministry of Transport, Public Wprks and Water Management, Delft, The Netherlands, 2009.

[4] A. Kneepkens, T. van Hoof, H. Schaefer, and W. van Keulen, VIA-RAL for Porous Asphalt: A Result of research and Development, But Most of all of implementation, Wegbouwkundige Werkdagen, Delft, The Netherlands, 2004.

[5] X. H. Liu and F. Civian, "Characterization and prediction of formation damage in two-phase flow systems," in Proceedings of the SPE Production Operations Symposium, Oklahoma City, Okla, USA, 1993.

[6] X. Liu and F. Civan, "Multiphase mud fluid infiltration and filter cake formation model," in Proceedings of the SPE International Symposium on Oilfield Chemistry, pp. 607-621, March 1989.

[7] X. Liu and C. Farouk, "Formation damage and skin factor due to filter cake formation and fines migration in the nearwellbore region," in Proceedings of the International Symposium on Formation Damage Control, pp. 259-273, Lafayette, Ind, USA, February 1994.

[8] C. Gruesbeck and R. E. Collins, "Entrainment and deposition of fines particles in porous media," Society of Petroleum Engineers Journal, vol. 22, no. 6, pp. 847-856, 1982.

[9] M. F. El-Amin, A. Salama, and S. Sun, "Numerical and dimensional investigation of two-phase countercurrent imbibition in porous media," Journal of Computational and Applied Mathematics, vol. 242, pp. 285-296, 2013.

[10] S. Sun, A. Salama, and M. F. El Amin, "Matrix-oriented implementation for the numerical solution of the partial differential equations governing flows and transport in porous media," Computers \& Fluids, vol. 68, pp. 38-46, 2012.

[11] M. El-Amin, A. Salama, and S. Sun, "Effects of gravity and inlet location on a two-phase countercurrent imbibition in porous media," International Journal of Chemical Engineering, vol. 2012, Article ID 210128, 7 pages, 2012.

[12] M. F. El-Amin and S. Sun, "Effects of gravity and inlet/outlet location on a two-phase cocurrent imbibition in porous media," Journal of Applied Mathematics, vol. 2011, Article ID 673523, 18 pages, 2011.

[13] R. D. Skeel and M. Berzins, "A method for the spatial discretization of parabolic equations in one space variable," SIAM Journal on Scientific and Statistical Computing, vol. 11, no. 1, pp. 1-32, 1990. 


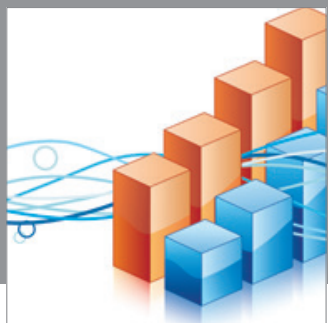

Advances in

Operations Research

mansans

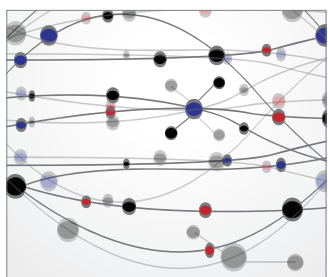

The Scientific World Journal
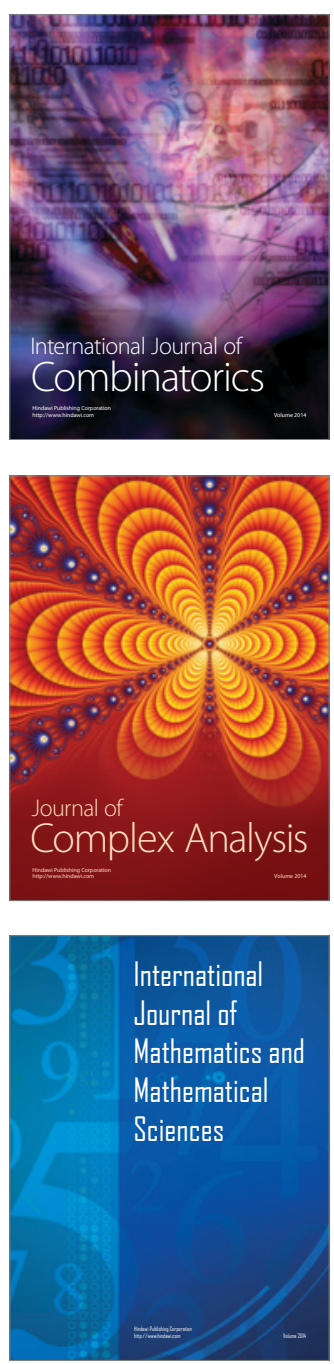
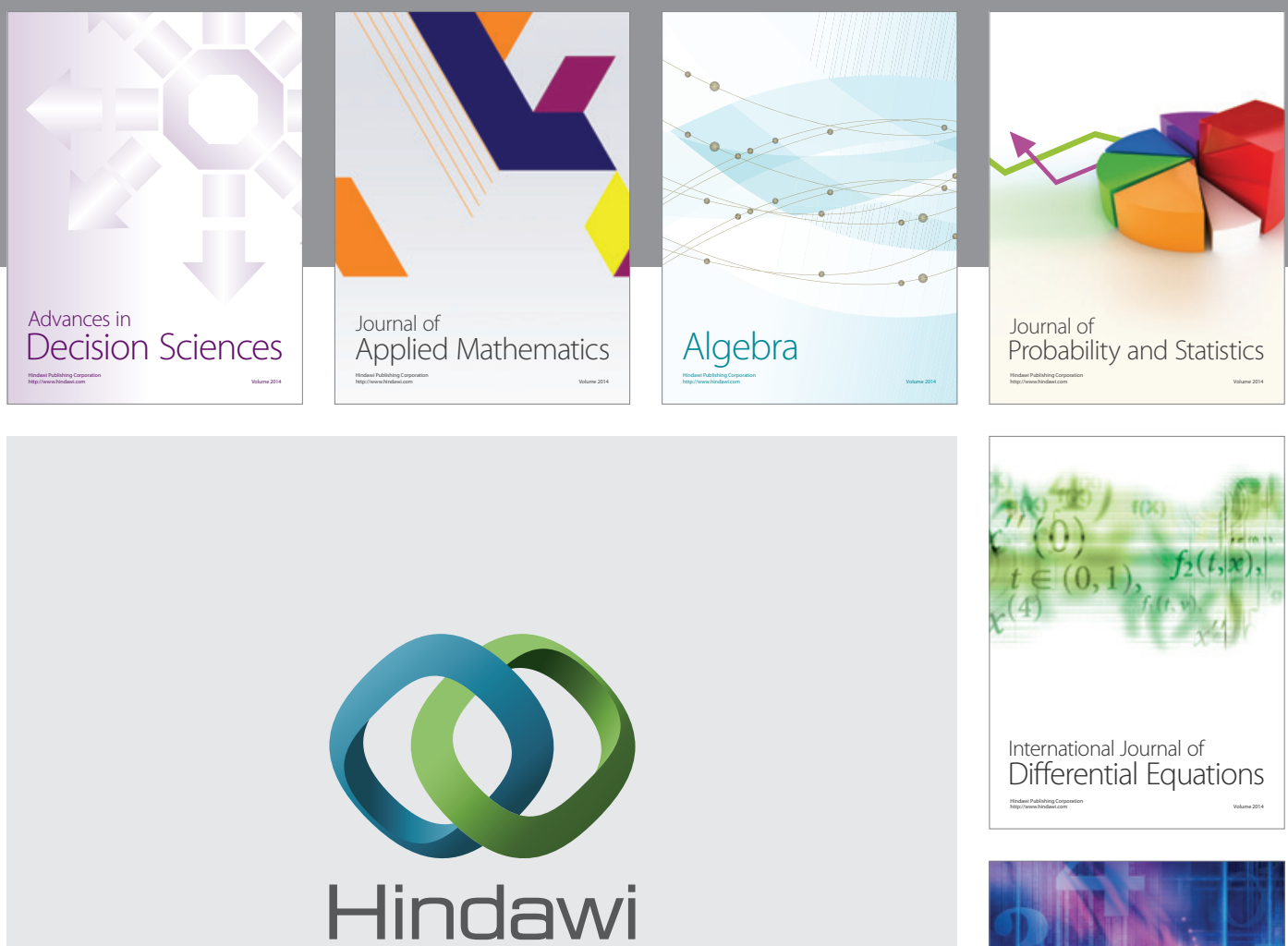

Submit your manuscripts at http://www.hindawi.com
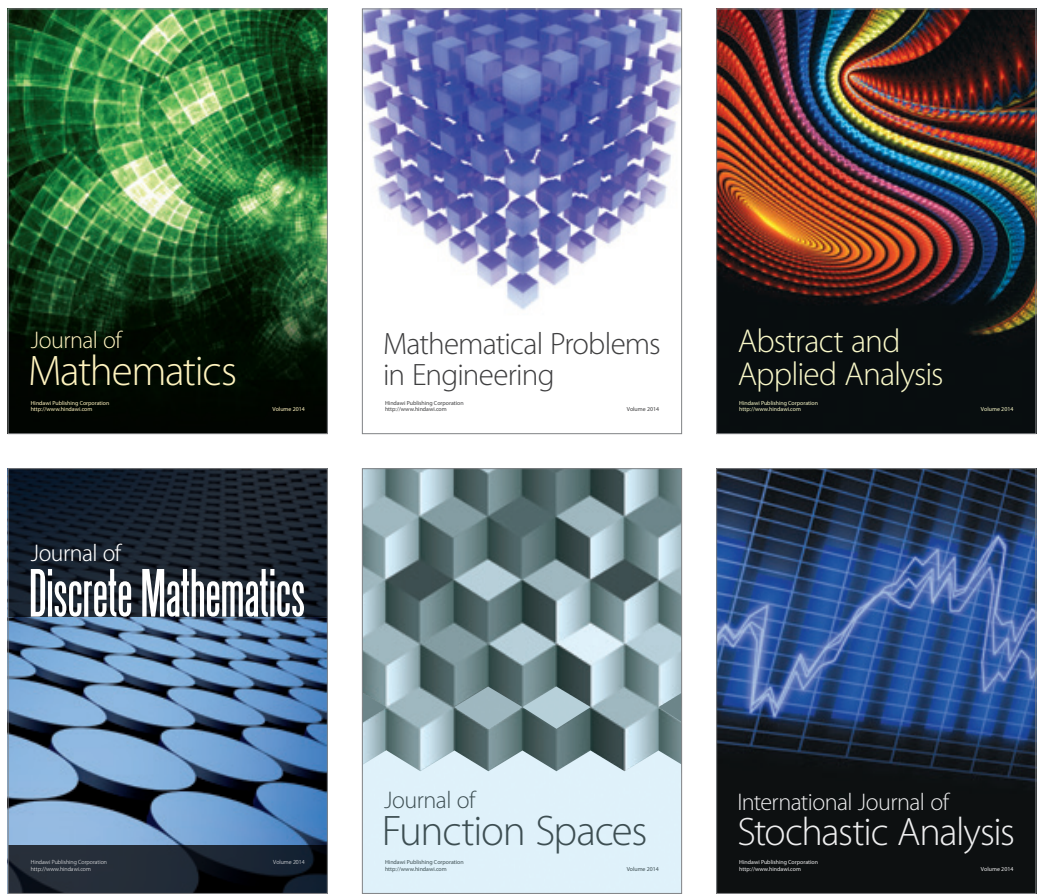

Journal of

Function Spaces

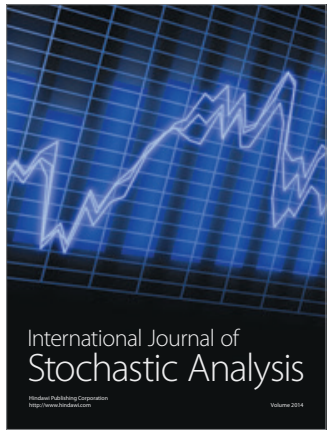

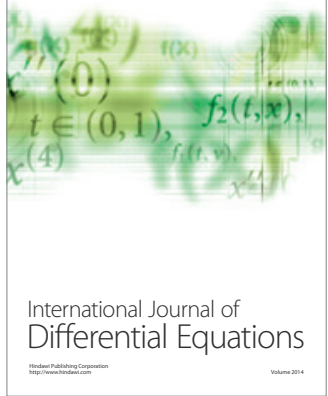
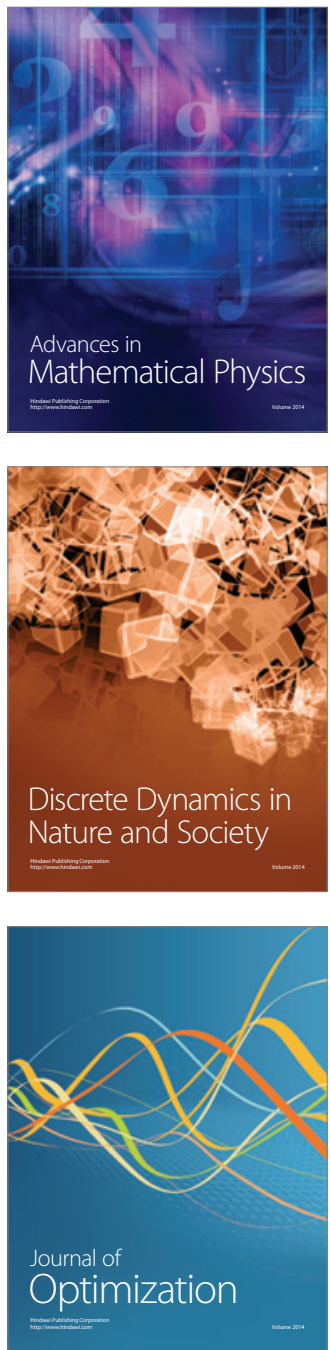